\title{
A Class of Estimators for Population Variance in Two Occasion Rotation Patterns
}

\author{
G. N. Singh ${ }^{1, a}$, Priyanka ${ }^{b}$, Shakti Prasad ${ }^{a}$, Sarjinder Singh ${ }^{c}$, Jong-Min Kim $^{d}$ \\ ${ }^{a}$ Department of Applied Mathematics, Indian School of Mines \\ ${ }^{b}$ Department of Mathematics, Shivaji College, University of Delhi \\ ${ }^{c}$ Department of Mathematics, Texas A \& M University - Kingsville \\ ${ }^{d}$ Division of Science and Mathematics, University of Minnesota at Morris
}

\begin{abstract}
A variety of practical problems can be addressed in the framework of rotation (successive) sampling. The present work presents a sample rotation pattern where sampling units are drawn on two successive occasions. The problem of estimation of population variance on current (second) occasion in two - occasion successive (rotation) sampling has been considered. A class of estimators has been proposed for population variance that includes many estimators as a particular case. Asymptotic properties of the proposed class of estimators are discussed. The proposed class of estimators is compared with the sample variance estimator when there is no matching from the previous occasion. Optimum replacement policy is discussed. Results are supported with the empirical means of comparison.
\end{abstract}

Keywords: Successive (rotation) sampling, variance estimation, bias, mean square error, optimum replacement policy.

\section{Introduction}

A variety of practical problems can fall in the arena of applied and environmental sciences where various characters opt to change with respect to different parameters; such changes are inherent behavior of the nature. Some type of changes directly or indirectly affect the quality of human living and surrounding. Such changes draw the attention of human intelligentsia to know the pattern (or the rate of change) over a desired period or to know the amount of change at any given point of time (occasion) or simultaneously to know both situations. This requires the continuous monitoring of real life situations. If the situations are required to be monitored are concerned with a very large group of individuals, it is difficult, time taking and a costly affair. For example an investigator or owner of a cold drink industry may be interested in the following type of problems: (a) The average or total sales of cold drinks for the current season, (b) The change in average sale of cold drinks for two different seasons, or (c) Simultaneously to know both (a) and (b).

The follow-up of objectives are conducted by means of sampling on successive occasions (over years or seasons or months) according to a specific rule, with the partial replacement of units, called successive (rotation) sampling. The problem of successive (rotation) sampling with a partial replacement of sampling units was first considered by Jessen (1942) in the analysis of survey which collected

\footnotetext{
Authors are also thankful to the Indian School of Mines, Dhanbad for providing financial assistance to carry out the present research work.

${ }^{1}$ Corresponding author: Professor, Department of Applied Mathematics, Indian School of Mines, Dhanbad-826004, India. E-mail: gnsingh_ism@yahoo.com
} 
farm data. He pioneered using the entire information collected in previous investigations (occasions). The theory of successive (rotation) sampling was further extended by Patterson (1950), Rao and Graham (1964), Gupta (1979), Das (1982) and Chaturvedi and Tripathi (1983), among others. Sen (1971) applied this theory with success in designing the estimator for the population mean on the current occasion using information on two auxiliary variables available from previous occasions. Sen (1972, 1973) extended his work for several auxiliary variables. Singh et al. (1991) and Singh and Singh (2001) used the auxiliary information from the current occasion and proposed estimators for the current population mean in two-occasion successive (rotation) sampling. Singh (2003) extended their work for h-occasions successive sampling. Feng and Zou (1997) and Biradar and Singh (2001) used the auxiliary information on both the occasions to estimate the current population mean in successive sampling.

In many situations, information on an auxiliary variable may be readily available on the first as well as on the second occasion. For example, the tonnage (or seat capacity) of each vehicle or ship is known in survey sampling of transportation, number of beds in different hospitals may be known in hospital surveys, number of polluting industries and vehicles are known in environmental survey, nature of employment status, educational status, food availability and medical aids of a locality are well known in advance to estimate various demographic parameters in demographic surveys. Many other situations in biological (life) sciences could be explored to show the benefits of the present study. Utilizing the auxiliary information from both occasions, Singh (2005), Singh and Priyanka (2006, 2007, 2008), and Singh and Karna (2009) proposed a chain-type ratio, difference and regression estimators to estimate the population mean at current (second) occasion in two-occasion successive (rotation) sampling.

A large number of estimators that estimate the population mean on the current occasion have been proposed by various authors, however, only limited efforts have been made to estimate the population variance on the current occasion in two occasions successive (rotation) sampling.

The present work develops a one- parameter class of estimators that estimate the population variance on the current occasion in two-occasion successive (rotation) sampling. The proposition of the class of estimators is such that it includes many estimators as a particular case for particular values of the parameter. Following the work of Singh and Shukla (1987), we propose a one parameter class of estimators to estimate the population variance at current occasion in two-occasion rotation patterns. Bias and mean square error of the proposed class of estimators have been derived. Asymptotic properties are discussed. The proposed class of estimators is compared with the sample variance estimator when there is no matching. Optimum replacement policy is discussed. The results have been supported with suitable empirical studies.

\section{Notations}

Let $U=\left(U_{1}, U_{2}, \ldots, U_{N}\right)$ be the finite population of $N$ (large) units and which is assumed to remain unchanged over two occasions. Let $x(y)$ be the character under study on the first (second) occasion respectively. A simple random sample (without replacement) of size $n$ units is drawn on the first occasion and a random sub-sample of size $m=n \lambda$ units from the sample on the first occasion is retained (matched) for its use on the current (second) occasion. A fresh (un-matched) sample of size $u=(n-m)=n \mu$ units is drawn on the current occasion from the entire population by a simple random sampling (without replacement) method so that the sample size on the current occasion is also $n$. $\lambda$ and $\mu(\lambda+\mu=1)$ are the fractions of the matched and fresh samples, respectively, on the current occasion. Now, we consider the following notations for their further use: 
$S_{x}^{2}=(N-1)^{-1} \sum_{i=1}^{N}\left(x_{i}-\bar{X}\right)^{2}:$ The population mean square (variance) of variable $x$.

$S_{y}^{2}$ : The population mean square (variance) of variable $y$.

$s_{x(n)}^{2}$ : The sample mean square (variance) of variable $x$ and based on sample of size $n$ on the first occasion.

$s_{x(m)}^{2}, s_{y(m)}^{2}$ : The sample mean squares (variances) of variables $x$ and $y$ of the matched sample of size $m$ on the first and current (second) occasions respectively.

$s_{y(u)}^{2}$ : The sample mean square (variance) of variable $y$ based on the unmatched (fresh) sample of size $u$ on the current (second) occasion.

$f(=n / N)$ : The sampling fraction.

\section{Proposed Class of Estimators}

To estimate the population variance $S_{y}^{2}$ (Population mean square) on the current (second) occasion, two independent estimators are suggested. One is based on sample of size $u=(n \mu)$ drawn fresh on the current (second) occasion, and which is given by

$$
T_{1 u}=s_{y(u)}^{2}
$$

second estimator is a one-parameter class of estimators based on the sample of size $m=(n \lambda)$ common with both occasions and is defined as

$$
T_{2 m}(d)=s_{y(m)}^{2}\left[\frac{(A+C) s_{x(n)}^{2}+f B s_{x(m)}^{2}}{(A+f B) s_{x(n)}^{2}+C s_{x(m)}^{2}}\right],
$$

where $A=(d-1)(d-2), B=(d-1)(d-4), C=(d-2)(d-3)(d-4), f(=n / N)$ and $d$ is a non negative constant identified to minimize the mean square error of $T_{2 m}(d)$.

Now considering the convex linear combination of the estimators $T_{1 u}$ and $T_{2 m}(d)$, we have a class of estimators of $S_{y}^{2}$ as

$$
\hat{T}_{d}=\varphi T_{1 u}+(1-\varphi) T_{2 m}(d),
$$

where $\varphi$ is a unknown constant to be determined to achieve the minimum mean square error of the class of estimators $\hat{T}_{d}$.

Remark 1. The following estimators can be identified as a particular case of suggested class of estimators $\hat{T}_{d}$ to estimate the population variance on the current occasion in two-occasion successive (rotation) sampling for different values of the unknown parameter $d$.

(i) $\hat{T}_{1}=\varphi T_{1 u}+(1-\varphi) T_{2 m}(1)$,

(ii) $\hat{T}_{2}=\varphi T_{1 u}+(1-\varphi) T_{2 m}(2)$,

(iii) $\hat{T}_{3}=\varphi T_{1 u}+(1-\varphi) T_{2 m}(3)$,

(iv) $\hat{T}_{4}=\varphi T_{1 u}+(1-\varphi) T_{2 m}(4)$,

where $T_{2 m}(1)=s_{y(m)}^{2}\left[s_{x(n)}^{2} / s_{x(m)}^{2}\right], T_{2 m}(2)=s_{y(m)}^{2}\left[s_{x(m)}^{2} / s_{x(n)}^{2}\right], T_{2 m}(3)=s_{y(m)}^{2}\left[\left\{N s_{x(n)}^{2}-n s_{x(m)}^{2}\right\} /\{(N-\right.$ $\left.\left.n) s_{x(n)}^{2}\right\}\right]$ and $T_{2 m}(4)=s_{y(m)}^{2}$ are respectively ratio, product, dual to ratio and sample variance type estimators. 


\section{Bias and Mean Square Error of the Proposed Estimator $\hat{T}_{d}$}

The proposed class of estimators $\hat{T}_{d}$ defined in Equation (3.3) is also a biased estimator of $S_{y}^{2}$, so, its bias $B(\cdot)$ and mean square error $M(\cdot)$ up-to the first order of approximations is derived under large sample approximations with the following transformations:

$$
s_{y(m)}^{2}=S_{y}^{2}\left(1+e_{0(m)}\right), \quad s_{y(u)}^{2}=S_{y}^{2}\left(1+e_{0(u)}\right), \quad s_{x(m)}^{2}=S_{x}^{2}\left(1+e_{1(m)}\right), \quad s_{x(n)}^{2}=S_{x}^{2}\left(1+e_{1(n)}\right),
$$

such that $E\left(e_{0(m)}\right)=E\left(e_{0(u)}\right)=E\left(e_{1(m)}\right)=E\left(e_{1(n)}\right)=0$,

$$
\begin{aligned}
& E\left(e_{1(m)}^{2}\right)=\frac{\left\{\beta_{2}(x)-1\right\}}{m}, \quad E\left(e_{1(n)}^{2}\right)=\frac{\left\{\beta_{2}(x)-1\right\}}{n}, \quad E\left(e_{0(m)} e_{1(m)}\right)=\frac{(h-1)}{m}, \\
& E\left(e_{1(m)} e_{1(n)}\right)=\frac{\left\{\beta_{2}(x)-1\right\}}{n}, \quad E\left(e_{0(m)} e_{1(n)}\right)=\frac{(h-1)}{n},
\end{aligned}
$$

where $h=\left(\mu_{22} /\left(\mu_{20} \mu_{02}\right)\right), \mu_{r s}=1 / N \sum_{i=1}^{N}\left(y_{i}-\bar{Y}\right)^{r}\left(x_{i}-\bar{X}\right)^{s}$. Under the above transformations, the estimator $T_{2 m}(d)$ takes the following form:

$$
T_{2 m}(d)=S_{y}^{2}\left(1+e_{0(m)}\right)\left(1+d_{1} e_{1(n)}+d_{2} e_{1(m)}\right)\left(1+d_{3} e_{1(n)}+d_{4} e_{1(m)}\right)^{-1},
$$

where $d_{1}=(A+C) /(A+f B+C), d_{2}=(f B) /(A+f B+C), d_{3}=(A+f B) /(A+f B+C)$ and $d_{4}=$ $C /(A+f B+C)$.

Thus, we have the following theorems:

Theorem 1. The bias of the estimator $\hat{T}_{d}$ to the first order of approximations is obtained as

$$
B\left(\hat{T}_{d}\right)=(1-\varphi) B\left\{T_{2 m}(d)\right\}
$$

where

$$
\begin{aligned}
B\left\{T_{2 m}(d)\right\}= & {\left[\left\{\beta_{2}(x)-1\right\}\left\{\frac{1}{n}\left(-d_{1} d_{3}-d_{2} d_{3}-d_{1} d_{4}+d_{3}^{2}+2 d_{3} d_{4}\right)+\frac{1}{m}\left(d_{4}^{2}-d_{2} d_{4}\right)\right\}\right.} \\
& \left.+(h-1)\left\{\frac{1}{n}\left(d_{1}-d_{3}\right)+\frac{1}{m}\left(d_{2}-d_{4}\right)\right\}\right] S_{y}^{2} .
\end{aligned}
$$

Proof: The bias of the estimator $\hat{T}_{d}$ is given by

$$
\begin{aligned}
B\left(\hat{T}_{d}\right) & =E\left[\hat{T}_{d}-S_{y}^{2}\right]=\varphi E\left(T_{1 u}-S_{y}^{2}\right)+(1-\varphi) E\left(T_{2 m}(d)-S_{y}^{2}\right) \\
& =\varphi B\left(T_{1 u}\right)+(1-\varphi) B\left\{T_{2 m}(d)\right\} .
\end{aligned}
$$

Since, $T_{1 u}$ is unbiased for $S_{y}^{2}$, therefore,

$$
B\left(T_{1 u}\right)=0
$$

and

$$
\begin{aligned}
B\left(T_{2 m}(d)\right) & =E\left[T_{2 m}(d)-S_{y}^{2}\right] \\
& =\varphi E\left[S_{y}^{2}\left(1+e_{0(m)}\right)\left(1+d_{1} e_{1(n)}+d_{2} e_{1(m)}\right)\left(1+d_{3} e_{1(n)}+d_{4} e_{1(m)}\right)^{-1}-S_{y}^{2}\right] .
\end{aligned}
$$


Now expanding the right hand side of above expression binomially, taking expectations and retaining the terms up-to the first order of approximations, we have

$$
\begin{aligned}
B\left\{T_{2 m}(d)\right\}= & {\left[\left\{\beta_{2}(x)-1\right\}\left\{\frac{1}{n}\left(-d_{1} d_{3}-d_{2} d_{3}-d_{1} d_{4}+d_{3}^{2}+2 d_{3} d_{4}\right)+\frac{1}{m}\left(d_{4}^{2}-d_{2} d_{4}\right)\right\}\right.} \\
& \left.+(h-1)\left\{\frac{1}{n}\left(d_{1}-d_{3}\right)+\frac{1}{m}\left(d_{2}-d_{4}\right)\right\}\right] S_{y}^{2} .
\end{aligned}
$$

Using Equation (4.3) and Equation (4.4) in Equation (4.2), we get the bias of the estimator $\hat{T}_{d}$ as given in Equation (4.1).

Theorem 2. The mean square error of the estimator $\hat{T}_{d}$ is given by

$$
M\left(\hat{T}_{d}\right)=\varphi^{2} V\left(T_{1 u}\right)+(1-\varphi)^{2} M\left\{T_{2 m}(d)\right\}_{o p t},
$$

where

$$
\begin{aligned}
V\left(T_{1 u}\right) & =\frac{1}{u}\left\{\beta_{2}(y)-1\right\} S_{y}^{4}, \\
M\left\{T_{2 m}(d)\right\}_{o p t} & =\left[\frac{a}{m}+\left(\frac{1}{m}-\frac{1}{n}\right) b\right] S_{y}^{4},
\end{aligned}
$$

where $a=\left\{\beta_{2}(y)-1\right\}, b=\alpha^{*^{2}}\left\{\beta_{2}(x)-1\right\}+2 \alpha^{*}(h-1), \alpha^{*}=[\alpha]_{d=d_{0}}, \alpha=\left(d_{2}-d_{4}\right)=\left(d_{3}-d_{1}\right)$ and $d_{0}$ is the optimum value of $d$.

Proof: Since $T_{1 u}$ and $T_{2 m}(d)$ are based on two independent samples, therefore, for large $N$ the covariance type term is ignored. Hence

$$
\begin{aligned}
M\left(\hat{T}_{d}\right) & =E\left[\hat{T}_{d}-S_{y}^{2}\right]^{2}=E\left[\varphi\left(T_{1 u}-S_{y}^{2}\right)+(1-\varphi)\left(T_{2 m}(d)-S_{y}^{2}\right)\right]^{2} \\
& =\varphi^{2} V\left(T_{1 u}\right)+(1-\varphi)^{2} M\left\{T_{2 m}(d)\right\}
\end{aligned}
$$

where

$$
\begin{aligned}
V\left(T_{1 u}\right) & =E\left[T_{1 u}-S_{y}^{2}\right]^{2}=\frac{1}{u}\left\{\beta_{2}(y)-1\right\} S_{y}^{4}, \\
M\left(T_{2 m}(d)\right) & =E\left[T_{2 m}(d)-S_{y}^{2}\right]^{2} \\
& =\varphi E\left[S_{y}^{2}\left(1+e_{0(m)}\right)\left(1+d_{1} e_{1(n)}+d_{2} e_{1(m)}\right)\left(1+d_{3} e_{1(n)}+d_{4} e_{1(m)}\right)^{-1}-S_{y}^{2}\right]^{2} .
\end{aligned}
$$

Now expanding the right hand side of above expression binomially, taking expectations, retaining the terms up-to the first order of approximations and for large $N$ ignoring fpc, we have

$$
M\left\{T_{2 m}(d)\right\}=\left[\frac{\left\{\beta_{2}(y)-1\right\}}{m}+\left(\frac{1}{m}-\frac{1}{n}\right) \alpha^{2}\left\{\beta_{2}(x)-1\right\}+2 \alpha(h-1)\right] S_{y}^{4} .
$$

The above expression is a function of $\alpha$, which is a function of $d$, that can be minimized for $d$; therefore, we have $M\left\{T_{2 m}(d)\right\} / \partial d=0$, this gives

$$
\alpha=\frac{f B-C}{A+f B+C}=\frac{(1-h)}{\left\{\beta_{2}(x)-1\right\}} ; \text { assuming } \quad \frac{\partial \alpha}{\partial d} \neq 0 .
$$


A close look of the Equation (4.8) reveals that it is a cubic equation in $d$. Therefore, for given $h$ and $\beta_{2}(x)$ one will get three optimum values of $d$ for which $M\left\{T_{2 m}(d)\right\}$ attains the same minimum value. The possibility of getting negative or imaginary roots cannot be ruled out. However, Shukla (1988) has pointed out that for any choice of $f, h$ and $\beta_{2}(x)$, there exists at least one positive real root of the Equation (4.8) ensuring that $M\left\{T_{2 m}(d)\right\}$ attains its minimum within the parametric space $(0, \infty)$. Since, there may exists at most three optimum values of $d$, a criterion for selecting suitable value of optimum $d$ may be set as follows:

"Out of all the possible values of optimum $d$, select the $d=d_{0}$ as the most suitable choice, which makes $\left|B\left\{T_{2 m}(d)\right\}\right|$ smallest".

Hence, the minimum mean square error of $T_{2 m}(d)$ is given by

$$
M\left\{T_{2 m}(d)\right\}_{o p t}=\left[\frac{a}{m}+\left(\frac{1}{m}-\frac{1}{n}\right) b\right] S_{y}^{4},
$$

where $a=\left\{\beta_{2}(y)-1\right\}, b=\alpha^{*^{2}}\left\{\beta_{2}(x)-1\right\}+2 \alpha^{*}(h-1), \alpha^{*}=[\alpha]_{d=d_{0}}$.

Using Equation (4.8) and Equation (4.9) in Equation (4.6) we get the expression for the mean square error of $\hat{T}_{d}$ as given in Equation (4.5).

Remark 2. The bias and mean square error of the estimators $\hat{T}_{1}, \hat{T}_{2}, \hat{T}_{3}$ and $\hat{T}_{4}$ discussed in remark 1 can be deduced from Equation (4.1) and Equation (4.5) for specific values of the parameter $d$ (i.e., $d=$ $1,2,3,4)$.

Theorem 3. The estimator $T_{2 m}(d)$, its bias and mean square error are asymptotically convergent to the estimator $T_{2 m}(1)$, its bias and mean square error respectively for large $d$.

Proof: Since, $d \neq 0$, dividing numerator and denominator of the R. H. S of Equation (3.2) by $d^{3}$ and taking the limit as $d \rightarrow \infty$, we have

$$
\lim _{d \rightarrow \infty} T_{2 m}(d)=s_{y(m)}^{2}\left[\frac{s_{x(n)}^{2}}{s_{x(m)}^{2}}\right]=T_{2 m}(1) .
$$

This is the ratio type estimator to estimate population variance given by Isaki (1983). Similarly, using the expression of bias and mean square error of the estimator $T_{2 m}(d)$, it is easy to see that

$$
\lim _{d \rightarrow \infty} B\left\{T_{2 m}(d)\right\}=B\left\{T_{2 m}(1)\right\}
$$

and

$$
\lim _{d \rightarrow \infty} M\left\{T_{2 m}(d)\right\}=M\left\{T_{2 m}(1)\right\}
$$

The proposed class of estimators converges to a well-defined estimator even if one chooses arbitrarily a larger value of the unknown parameter $d$. The bias and mean square error also tends asymptotically to that of Isaki (1983) type estimator to estimate finite population variance. There is no need to bother about the existence of the estimator defined for the matched portion of the sample while choosing a larger value of $d$. 


\section{Minimum Mean Square Error of the Proposed Estimator $\hat{T}_{d}$}

Since, mean square error of $\hat{T}_{d}$ in Equation (4.5) is a function of unknown constant $\varphi$, therefore, it is minimized with respect to $\varphi$ and subsequently the optimum value of $\varphi$ is obtained as

$$
\varphi_{o p t}=\frac{M\left\{T_{2 m}(d)\right\}_{o p t}}{V\left(T_{1 u}\right)+M\left\{T_{2 m}(d)\right\}_{o p t}}
$$

and substituting the value of $\varphi_{\text {opt }}$ from Equation (5.1) in Equation (4.5), we get the optimum mean square error of the estimator $\hat{T}_{d}$ as

$$
M\left(\hat{T}_{d}\right)_{o p t}=\frac{V\left(T_{1 u}\right) \cdot M\left\{T_{2 m}(d)\right\}_{o p t}}{V\left(T_{1 u}\right)+M\left\{T_{2 m}(d)\right\}_{o p t}} .
$$

Further, to substitute the values from Equation (4.7) and Equation (4.9) in Equation (5.2), we get the simplified values of $M\left(\hat{T}_{d}\right)_{o p t}$ which is shown in Theorem 4.

Theorem 4. The minimum mean square error of the estimator $\hat{T}_{d}$ is derived as

$$
M\left(\hat{T}_{d}\right)_{o p t}=\frac{a}{n}\left[\frac{a+\mu b}{a+\mu^{2} b}\right] S_{y}^{4},
$$

where $\mu(=u / n)$ is the fraction of fresh sample drawn on the current (second) occasion. Again $M\left(\hat{T}_{d}\right)_{o p t}$ derived in Equation (5.3) is the function of $\mu$. To estimate the population variance on each occasion the better choice of $\mu$ is 1 (case of no matching); however, to estimate the change in variance from one occasion to the other, $\mu$ should be 0 (case of complete matching). But intuition suggests that an optimum choice of is desired to devise the amicable strategy for both problems simultaneously. Hence, in the next section of this problem, an optimum replacement policy for $\mu$ has been discussed.

\section{Optimum Replacement Policy}

The key design parameter affecting the estimates of change is the overlap between successive samples. Maintaining high overlap between repeats of a survey is operationally convenient, since many sampled units have been located and have some experience in the survey. Hence, to determine the optimum value of $\mu$ (fraction of sample to be drawn fresh on the current occasion) so that $S_{y}^{2}$ may be estimated with maximum precision, we minimize $M\left(\hat{T}_{d}\right)_{o p t}$ in (5.3) with respect to $\mu$ and hence we get

$$
\hat{\mu}=\frac{-a \pm \sqrt{a(a+b)}}{b}
$$

The real values of $\hat{\mu}$ exist, iff $a(a+b) \geq 0$. For any situation, which satisfies this condition, two real values of $\hat{\mu}$ are possible, hence, to choose a value of $\hat{\mu}$, it should be remembered that $0 \leq \hat{\mu} \leq 1$, all other values of $\hat{\mu}$ are inadmissible. If both the real values of $\hat{\mu}$ are admissible, the lowest one will be the best choice as it reduces the cost of the survey. Substituting the admissible value of $\hat{\mu}$ say $\mu_{0}$ from Equation (6.1) in Equation (5.3), we have the optimum values of the mean square error of the estimator $\hat{T}_{d}$, which is shown as

$$
M\left(\hat{T}_{d}\right)_{o p t^{*}}=\frac{a}{n}\left[\frac{a+\mu_{0} b}{a+\mu_{0}^{2} b}\right] S_{y}^{4} .
$$




\section{Efficiency Comparison}

To study the performance of the estimator $\hat{T}_{d}$, the percent relative efficiencies of the estimator $\hat{T}_{d}$ with respect to $s_{y(n)}^{2}$ (the natural estimator of $S_{y}^{2}$ ), when there is no matching. Since, $s_{y(n)}^{2}$ is unbiased estimator of $S_{y}^{2}$, so the variance of $s_{y(n)}^{2}$ for large $N$ (i.e., $N \rightarrow \infty$ ) is given by

$$
V\left(s_{y(n)}^{2}\right)=\frac{1}{n}\left\{\beta_{2}(y)-1\right\} S_{y}^{4} .
$$

Table 3, shows the optimum value of $\mu$ i.e., $\mu_{0}$ and the percent relative efficiency $E$ of the estimator $\hat{T}_{d}$ (under optimal condition) with respect to $s_{y(n)}^{2}$, where

$$
E=\frac{V\left(s_{y(n)}^{2}\right)}{M\left(\hat{T}_{d}\right)_{o p t^{*}}} \times 100 .
$$

\section{Numerical Illustrations}

The various results obtained in previous sections are now examined with the help of two natural population sets of data.

Populations Source: [Free access to the data by the Statistical Abstracts of the United States]. The first case analyzes the variance of the infant mortality rates of US in the year 2002. The population consists of $N=51$ states. Further let $Y_{i}$ (study variable) be the infant mortality rates in the year 2002 and $X_{i}$ be the infant mortality rates in the year 2000 in the $i^{\text {th }}$ state of US.

Similarly, the second natural population estimates the variance of the abortion rate in different states during 2000. Here, also the population consists of $N=51$ states. Let $Y_{i}$ (study variable) denote the rates of abortions during 2000 and $X_{i}$ be the rate of abortions in the year 1992 in the $i^{\text {th }}$ state of US.

Based on the above description, the values of the different required parameters for both populations are:

\section{Population I:}

$$
\begin{aligned}
N & =51, \quad \bar{X}=22.6686, \quad \bar{Y}=17.4431, \quad \beta_{2}(x)=25.7309, \quad \beta_{2}(y)=9.1367, \\
\mu_{22} & =6.2904 \times 10^{5}, \quad \mu_{20}=125.5091, \quad \mu_{02}=338.2041 \quad \text { and } \quad S_{y}^{2}=128.0193 .
\end{aligned}
$$

\section{Population II:}

$$
\begin{aligned}
N & =51, \quad \bar{X}=7.1824, \quad \bar{Y}=7.1216, \quad \beta_{2}(x)=3.9212, \quad \beta_{2}(y)=2.8800, \\
\mu_{22} & =15.1769, \quad \mu_{20}=2.3703, \quad \mu_{02}=2.1564 \quad \text { and } \quad S_{y}^{2}=2.4177 .
\end{aligned}
$$

Now, solving the cubic equation in (4.8) for $d$, we have three values of $d$ for each given values of $f$. The mean square error will be same for all the three values of $d$. So, using the criterion set above, Table 1 shows the best choice of optimum value of $d$ for different values of $f$, for Population I and Table 2 shows the same for Population II.

\section{Interpretation of Results and Conclusion}

Tables 1, 2 and 3 show that for both the populations the real optimum values of $d$ always exist and the percent relative efficiency in both cases is appreciable when compared with the sample variance 
Table 1: Best choice of $d$ for Population I, for different values of $f$

\begin{tabular}{|c|c|c|c|c|}
\hline$f$ & $d$ & Bias & |Bias $\mid$ & $d_{0}$ \\
\hline \multirow{3}{*}{0.01} & 1.9333 & -11465.00 & 11465.00 & \\
\hline & 6.1106 & -10548.00 & 10548.00 & \\
\hline & 2.2579 & -10139.00 & 10139.00 & 2.2579 \\
\hline \multirow{3}{*}{0.02} & 1.8905 & -5797.10 & 5797.10 & \\
\hline & 6.1343 & -5286.50 & 5286.50 & \\
\hline & 2.3123 & -4922.40 & 4922.40 & 2.3123 \\
\hline \multirow{3}{*}{0.03} & 1.8572 & -3896.30 & 3896.30 & \\
\hline & 6.1583 & -3532.50 & 3532.50 & \\
\hline & 2.3570 & -3194.90 & 3194.90 & 2.3570 \\
\hline \multirow{3}{*}{0.04} & 1.8293 & -2941.00 & 2941.00 & \\
\hline & 6.1824 & -2655.50 & 2655.50 & \\
\hline & 2.3960 & -2336.00 & 2336.00 & 2.3960 \\
\hline \multirow{3}{*}{0.05} & 1.8052 & -2365.30 & 2365.30 & \\
\hline & 6.2068 & -2129.20 & 2129.20 & \\
\hline & 2.4311 & -1823.20 & 1823.20 & 2.4311 \\
\hline \multirow{3}{*}{0.06} & 1.7838 & -1980.00 & 1980.00 & \\
\hline & 6.2313 & -1778.30 & 1778.30 & \\
\hline & 2.4634 & -1482.90 & 1482.90 & 2.4634 \\
\hline \multirow{3}{*}{0.07} & 1.7644 & -1703.80 & 1703.80 & \\
\hline & 6.2560 & -1527.60 & 1527.60 & \\
\hline & 2.4933 & -1240.80 & 1240.80 & 2.4933 \\
\hline \multirow{3}{*}{0.08} & 1.7468 & -1496.00 & 1496.00 & \\
\hline & 6.2809 & -1339.60 & 1339.60 & \\
\hline & 2.5214 & -1060.10 & 1060.10 & 2.5214 \\
\hline
\end{tabular}

Table 2: Best choice of $d$ for Population II, for different values of $f$

\begin{tabular}{|c|c|c|c|c|}
\hline$f$ & $d$ & Bias & |Bias $\mid$ & $d_{0}$ \\
\hline \multirow{3}{*}{0.01} & 1.8586 & -18.5827 & 18.5827 & \\
\hline & 7.1196 & -17.9382 & 17.9382 & \\
\hline & 2.1419 & -16.8658 & 16.8658 & 2.1419 \\
\hline \multirow{3}{*}{0.02} & 1.8074 & -9.4090 & 9.4090 & \\
\hline & 7.1572 & -8.9864 & 8.9864 & \\
\hline & 2.2069 & -8.1758 & 8.1758 & 2.2069 \\
\hline \multirow{3}{*}{0.03} & 1.7699 & -6.3261 & 6.3261 & \\
\hline & 7.1950 & -6.0023 & 6.0023 & \\
\hline & 2.2579 & -5.3035 & 5.3035 & 2.2579 \\
\hline \multirow{3}{*}{0.04} & 1.7396 & -4.7753 & 4.7753 & \\
\hline & 7.2331 & -4.5102 & 4.5102 & \\
\hline & 2.3016 & -3.8763 & 3.8763 & 2.3016 \\
\hline \multirow{3}{*}{0.05} & 1.7137 & -3.8403 & 3.8403 & \\
\hline & 7.2714 & -3.6148 & 3.6148 & \\
\hline & 2.3404 & -3.0244 & 3.0244 & 2.3404 \\
\hline \multirow{3}{*}{0.06} & 1.6911 & -3.2143 & 3.2143 & \\
\hline & 7.3100 & -3.0177 & 3.0177 & \\
\hline & 2.3759 & -2.4590 & 2.4590 & 2.3759 \\
\hline \multirow{3}{*}{0.07} & 1.6709 & -2.7655 & 2.7655 & \\
\hline & 7.3488 & -2.5912 & 2.5912 & \\
\hline & 2.4086 & -2.0567 & 2.0567 & 2.4086 \\
\hline \multirow{3}{*}{0.08} & 1.6526 & -2.4278 & 2.4278 & \\
\hline & 7.3879 & -2.2713 & 2.2713 & \\
\hline & 2.4392 & -1.7561 & 1.7561 & 2.4392 \\
\hline
\end{tabular}


Table 3: Optimum value of $\mu$ and percent relative efficiency of $\hat{T}_{d}$ (under optimal condition) with respect to $s_{v(n)}^{2}$

\begin{tabular}{ccc}
\hline \hline Population & I & II \\
\hline$\mu_{0}$ & 0.8158 & 0.6485 \\
PRE & 167.17 & 129.69 \\
\hline \hline
\end{tabular}

estimator when there is no matching. The nice convergence property of the proposed class of estimators reinforces the importance of incorporation of unknown parameter in the structure of the estimator as the optimum value of the parameter is always possible. Under the given framework it is possible to reduce the bias of the estimator defined for the matched portion of the sample along with the minimization of the mean square error (m. s. e.), which is generally not possible in other class of estimators. The analytical and empirical results support the theoretical justification of the work. The estimation of population variance on successive occasions should be encouraged as there are numerous practical situations that require the estimate of variance at different points of time as the characters are time dependent. Hence, the proposed class of estimators should be recommended to the survey statisticians for their use.

\section{Acknowledgement}

The authors are thankful to the referee for their valuable and inspiring suggestions.

\section{References}

Biradar, R. S. and Singh, H. P. (2001). Successive sampling using auxiliary information on both occasions, Calcutta Statistical Association Bulletin, 51, 243-251.

Chaturvedi, D. K. and Tripathi, T. P. (1983). Estimation of population ratio on two occasions using multivariate auxiliary information, Journal of Indian Statistical Association, 21, 113-120.

Das, A. K. (1982). Estimation of population ratio on two occasions, Journal of the Indian Society of Agricultural Statistics, 34, 1-9.

Feng, S. and Zou, G. (1997). Sample rotation method with auxiliary variable, Communications in Statistics-Theory and Methods, 26, 1497-1509.

Gupta, P. C. (1979). Sampling on two successive occasions, Journal of Statistical Research, 13, 7-16.

Isaki, C. T. (1983). Variance estimation using auxiliary information, Journal of the American Statistical Association, 78, 117-123.

Jessen, R. J. (1942). Statistical Investigation of a Sample Survey for obtaining farm facts, Iowa Agricultural Experiment Station Research Bulletin, 304, 1-104, Ames, Iowa, USA.

Patterson, H. D. (1950). Sampling on successive occasions with partial replacement of units, Journal of the Royal Statistical Society, 12, 241-255.

Rao, J. N. K. and Graham, J. E. (1964). Rotation design for sampling on repeated occasions, Journal of the American Statistical Association, 59, 492-509.

Sen, A. R. (1971). Successive sampling with two auxiliary variables, Sankhya, 33, Series B, 371-378.

Sen, A. R. (1972). Successive sampling with $p(p \geq 1)$ auxiliary variables, The Annals of Mathematical Statistics, 43, 2031-2034.

Sen, A. R. (1973). Theory and application of sampling on repeated occasions with several auxiliary variables, Biometrics, 29, 381-385.

Shukla, D. (1988). On some factor-type estimators for population parameters in sample surveys, Unpublished Ph.D. Dissertation, Banaras Hindu University, Varanasi, India. 
Singh, G. N. (2003). Estimation of population mean using auxiliary information on recent occasion in h-occasion successive sampling, Statistics in Transition, 6, 523-532.

Singh, G. N. (2005). On the use of chain-type ratio estimator in successive sampling, Statistics in Transition, 7, 21-26.

Singh, G. N. and Karna, J. P. (2009). Estimation of population mean on current occasion in twooccasion successive sampling, METRON, 67, 69-85.

Singh, G. N. and Priyanka, K. (2006). On the use of chain-type ratio to difference estimator in successive sampling, IJAMAS, 5, (S06), 41-49.

Singh, G. N. and Priyanka, K. (2007). On the use of auxiliary information in search of good rotation patterns on successive occasions, Bulletin of Statistics and Economics, 1, (A07), 42-60.

Singh, G. N. and Priyanka, K. (2008). Search of good rotation patterns to improve the precision of estimates at current occasion, Communications in Statistics - Theory and Methods, 37, 337-348.

Singh, G. N. and Singh, V. K. (2001). On the use of auxiliary information in successive sampling, Journal of the Indian Society of Agricultural Statistics, 54, 1-12.

Singh, V. K. and Shukla, D. (1987). One parameter family of factor-type ratio estimators, Metron, $\mathbf{4 5}$, 1-2, 30, 273-283.

Singh, V. K., Singh, G. N. and Shukla, D. (1991). An efficient family of ratio-cum-difference type estimators in successive sampling over two occasions, Journal of Scientific Research, 41, 149159. 